\section{Michel Duguet Jean-François Riou}

\section{ADRESSES}

M. Duguet : professeur. Laboratoire d'enzy mologie des acides nucléiques, institut de génétique et microbiologie, Cnrs URA \354, bâtiment 400 , université Paris Sud, 91405 Orsay Cedex, France. J.-F. Riou docteur ès siences. Département de biologie centre de recherches de Vitry-Alfortville, Rhône-Poulenc Rorer SA, 94403 Vitry-sur

\title{
De la topologie de l'ADN aux médicaments antibiotiques et anticancéreux
}

Le contrôle de la structure topologique de l'ADN et de nombreuses fonctions liées à son métabolisme (transcription, réplication, recombinaison...) s'effectue grâce à des enzymes spécialisées, les ADN topo-isomérases. Elles réalisent des coupures transitoires dans l'ADN et font passer des segments d'ADN à travers ces coupures avant de les refermer. Les topo-isomérases de type I produisent des coupures simple-brin alors que les topo-isomérases de type II coupent simultanément les deux brins de l'ADN. Ces enzymes sont les cibles intracellulaires de plusieurs classes d'agents possédant des propriétés antitumorales ou antibiotiques. On entrevoit aujourd'hui les mécanismes complexes expliquant les processsus qui vont de la production de lésions dans l'ADN jusqu'à la mort cellulaire, ouvrant des perspectives prometteuses dans la mise au point de nouveaux agents antitumoraux, anti-bactériens, et antiviraux plus sélectifs et plus performants.

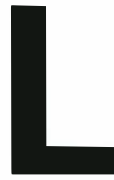

'existence des topo-isomérases est directement liée à la structure de l'ADN, dans laquelle les deux brins sont enlacés en une double hélice plectonémique (Watson-Crick). Tant que l'ADN est linéaire et que sa taille ne dépasse pas quelques $k b$, cette structure ne pose pas de problèmes particuliers. Au contraire, comment imaginer qu'un ADN génomique de plusieurs millions de kb (plus d'un mètre de longueur), compacté dans les chromosomes, puisse être décondensé, répliqué et recondensé en quelques heures sans se trouver irrémédiablement enchevêtré ?

Deux possibilités s'offraient à la nature pour résoudre ce problème : soit la structure de l'ADN devait être modifiée en faveur d'une structure côte à côte (paranémique) où les deux brins ne sont jamais enchevêtrés*, soit un mécanisme enzymatique très efficace permettait de supprimer les enchevêtrements (barrières topologiques). C'est cette dernière possibilité qui a prévalu avec "l'entrée en scène " des topo-isomérases. Ces enzymes sont, comme le fait remarquer Wang, des prestidigitateurs, dans la mesure où elles sont capables de faire passer un brin ou

* Des structures côte à côte de l'ADN, compatibles avec les données de diffraction, ont été effectivement imaginées dans les années 1977-1978, puis abandonnées. 


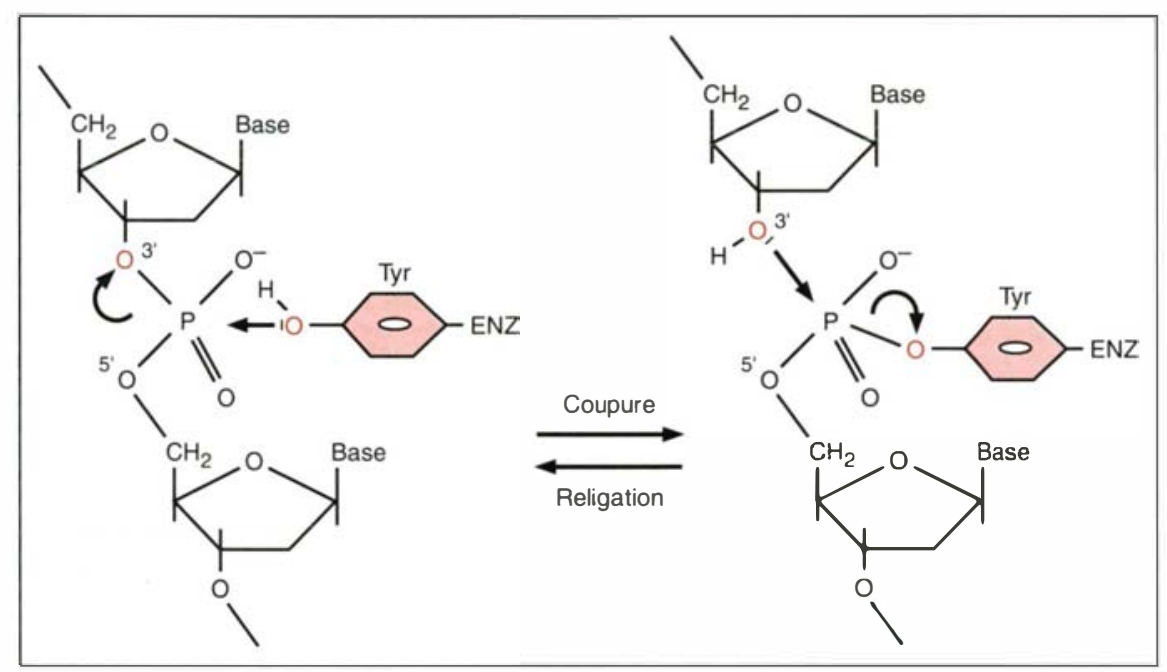

Figure 1. La réaction de trans-estérification caractérisant l'activité topo-isomérase. Le site actif de l'enzyme, une tyrosine, réagit par son groupement $\mathrm{OH}$ avec la liaison phosphodiester de I'ADN. Cette réaction, une trans-estérification, provoque une coupure du brin d'ADN et la formation d'un intermédiaire covalent entre la tyrosine de l'enzyme et une des extrémités (ici l'extrémité $5^{\prime}$ ) du brin d'ADN. La réaction inverse reforme la liaison phosphodiester et libère la tyrosine. Ainsi, la réaction de coupure/refermeture ne nécessite pas d'apport d'énergie.

un segment d'ADN à travers un autre sans laisser la moindre trace $[1,2]$.

La solution des topo-isomérases pour résoudre les problèmes d'enchevêtrement de l'ADN présente en outre un avantage considérable: ces enzymes, en modifiant le nombre d'enlacements entre les brins de la double hélice, sont capables de produire une forme activée de l'ADN, l'ADN surenroulé [3]. De manière un peu schématique, on peut considérer que c'est cette forme d'ADN qui permet d'initier des processus comme la réplication, la transcription ou la recombinaison.

Depuis la découverte chez $E$. coli, en 1971, de la première topo-isomérase,

d'inhibiteurs découvertes à ce jour. Une attention particulière sera portée aux mécanismes permettant d'expliquer l'activité antibiotique ou antitumorale de ces inhibiteurs, ainsi que les perspectives scientifiques et thérapeutiques envisageables dans un avenir proche.

\section{Les topo-isomérases produisent des coupures transitoires dans I'ADN}

La seule façon de réaliser le tour de magie qui consiste à faire passer un fragment d'ADN au travers d'un autre est d'effectuer une coupure transitoire dans l'ADN. L'opération se fait en trois temps: coupure de l'ADN, passage d'un autre segment à travers la coupure, refermeture de la brèche. Toutes les topo-isomérases fonctionnent sur ce principe général. Cependant, on distingue deux grands types de topo-isomérases: les enzymes de type I, capables de catalyser l'ouverture et la refermeture d'un seul brin d'ADN, et qui sont monomériques; les enzymes de type II, capables de catalyser l'ouverture et la refermeture simultanée ou séquentielle des deux brins de la molécule d'ADN et qui sont des protéines multimériques $[2,4]$.

Sur le plan chimique (figure 1), il s'agit d'une réaction de trans-estérification réversible conduisant à un intermédiaire covalent entre une tyrosine de l'enzyme et l'une des extrémités 5' ou 3' du brin d'ADN. La figure 2 montre un cycle de topoisomérisation pour la topo-isomérase II eucaryote [5]. Dans ce cas, l'enzyme est dimérique, chaque brin de l'ADN étant clivé par une sous-unité. En outre, la réaction nécessite la fixation et l'hydrolyse de l'ATP. Les intermédiaires clivés, dénommés aussi " complexes de clivage ", ont normalement une durée de vie très courte, le temps du passage de l'ADN à travers la coupure. Cette durée peut être considérablement augmentée par divers agents (antibiotiques, antitumoraux) interférant notamment avec la réaction de refermeture.

\section{Les topo-isomérases :} une famille complexe

Au fil de la découverte de nouvelles 


\section{RÉFÉRENCES}

1. Wang JC. Les enzymes qui modifient la topologie de l'ADN. Pour la Science 1982 ; 59: 56-71.

2. Wang JC. DNA topoisomerases. Annu Rev Biochem 1985 ; 54 : 665-97.

3. Bauer WR, Crick FHC, White J. L'ADN sous forme surenroulée. Pour la Science $1980 ; 35: 110-22$.

4. Wang JC. Recent studies of DNA topoisomerases. Biochim Biophys Acta 1987; 909 : $1-9$.

5. Osheroff N, Zechiedrich L, Gale KC. Catalytic function of DNA topoisomerase. Bio Essays 1991 ; 13 : 269-74.

6. Confalonieri F, Elie C, Nadal M, Bouthier de la Tour C, Forterre P, Duguet M. Reverse gyrase : a helicase-like domain and a type I topoisomerase in the same polypeptide. Proc Natl Acad Sci USA 1993; $90: 4753-7$.

7. Douc-Rasy S, Kayser A, Riou JF, Riou G. An ATP independent type II topoisomerase from trypanosomes. Proc Natl Acad Sci USA $1986 ; 83: 7152-6$.

8. Wang JC. DNA topoisomerases: why so many? J Biol Chem 1991; 266 : 6659-62.

9. Caron PR, Wang JC. DNA topoisomerases as targets of therapeutics : a structural overview. In : Andoh T, Ikeda M, Oguro M eds. Molecular biology of DNA topoisomerases and its application to chemotherapy. Boca Raton : CRC Press, 1993 : 1-18.

10. Tse-Dinh YC, Wang JG. Complete nucleotide sequence of the topA gene encoding Escherichia coli DNA topoisomerase I. J Mol Biol 1986; $191: 321-31$.

11. Wallis JW, Chrebet G, Brodsky G Rolfe M, Rothstein R. A hyper-recombination mutation in $S$. cerevisiae identifies a novel eukaryotic topoisomerase. Cell 1989 ; 58: 409-19.

12. Di Gate RJ, Marians KJ. Molecular cloning and DNA sequence analysis of Escherichia coli topB, the gene encoding topoisomerase III. I Biol Chem 1989; 264: $17924-30$

13. Bouthier de la Tour C, Portemer C, Huber R, Forterre P, Duguet M, Reverse gyrase in thermophilic eubacteria. $J$ Bacteriol 1991 ; 173 : $3921-3$

14. Slesarev A, Stetter KO, Lake JA, Gellert M, Krah R, Kozyavkin SA. DNA topoisomerase $\mathrm{V}$ is a relative of eukaryotic topoisomerase I from a hyperthermophilic

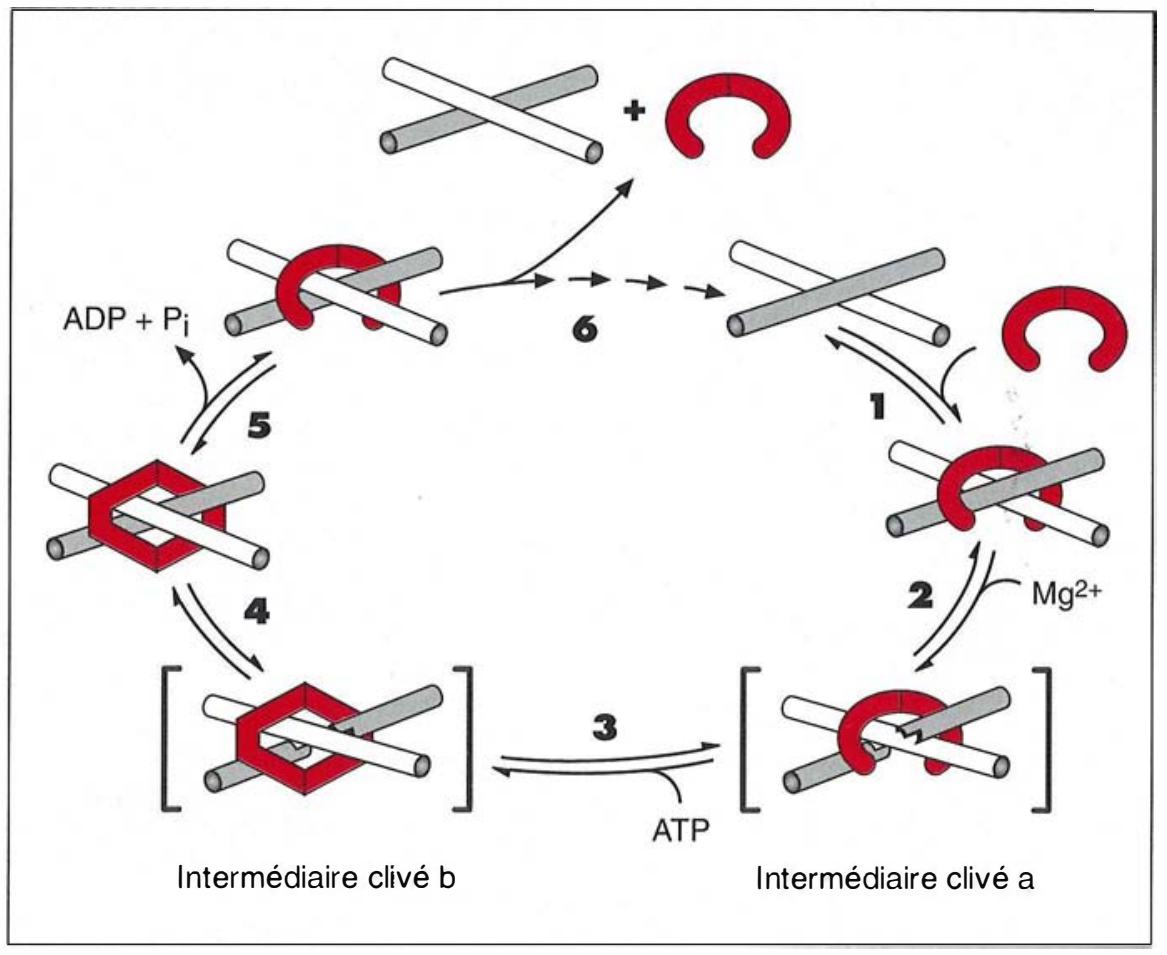

Figure 2. Les différentes étapes du cycle catalytique de la topo-isomérase II. La topo-isomérase II eucaryote (en rouge), constituée d'un homodimère, forme un complexe non covalent au niveau d'un croisement de deux doubles brins $d^{\prime} A D N$, dont l'un (en grisé) porte une séquence consensus dégénérée (étape 1 : fixation). Les deux brins de ce dernier sont clivés respectivement par chacune des deux sous-unités de la topo-isomérase II, grâce à deux réactions de trans-estérification (voir figure 1) produisant l'intermédiaire clivé a (étape 2: clivage de l'ADN). Le double brin d'ADN intact passe au travers de la brèche, sans doute grâce à un changement conformationnel de l'enzyme qui a fixé deux molécules d'ATP létape 3 : passage de brin). L'intermédiaire clivé $b$ reforme les liaisons phosphodiester, libère les tyrosines et la topo-isomérase reste fixée sur I'ADN sous forme de complexe non covalent (étape 4 : religation). L'ATP est hydrolysé, ce qui régénère une forme active de l'enzyme qui se dissocie de I'ADN (étape 5: régénération de l'enzyme). Chaque cycle catalytique modifie d'une valeur de deux le nombre de liens topologiques d'une molécule $d^{\prime} A D N$ circulaire. (Adapté avec l'aimable permission de N. Osheroff [5].)

topo-isomérases, la nomenclature de ces enzymes devient plus touffue (figure 3). La distinction entre topoisomérases de type I et de type II sur la base des coupures transitoires, simple-brin dans le premier cas, double-brin dans le second cas, est maintenant solidement établie $[2,4]$. Au contraire, le critère de dépendance vis-à-vis de l'ATP ne doit plus être utilisé pour distinguer ces deux types d'enzymes, car il souffre au moins deux exceptions: les gyrases inverses sont des topo-isomérases I dépendantes de l'ATP [6], certaines topo-isomérases II sont indépendantes de l'ATP [7].

\section{Topo-isomérases de type I}

Ces enzymes, généralement monomériques, apparaissent très hétéroclites et peuvent être divisées en au moins trois groupes $[2,4,8,9]$ : - celui représenté par les produits du gène TopA chez $E$. coli [10], qui comprennent principalement des topo-isomérases bactériennes, mais aussi la protéine codée par un gène de levure ( $S_{c}$ Top 3) dont la séquen- 


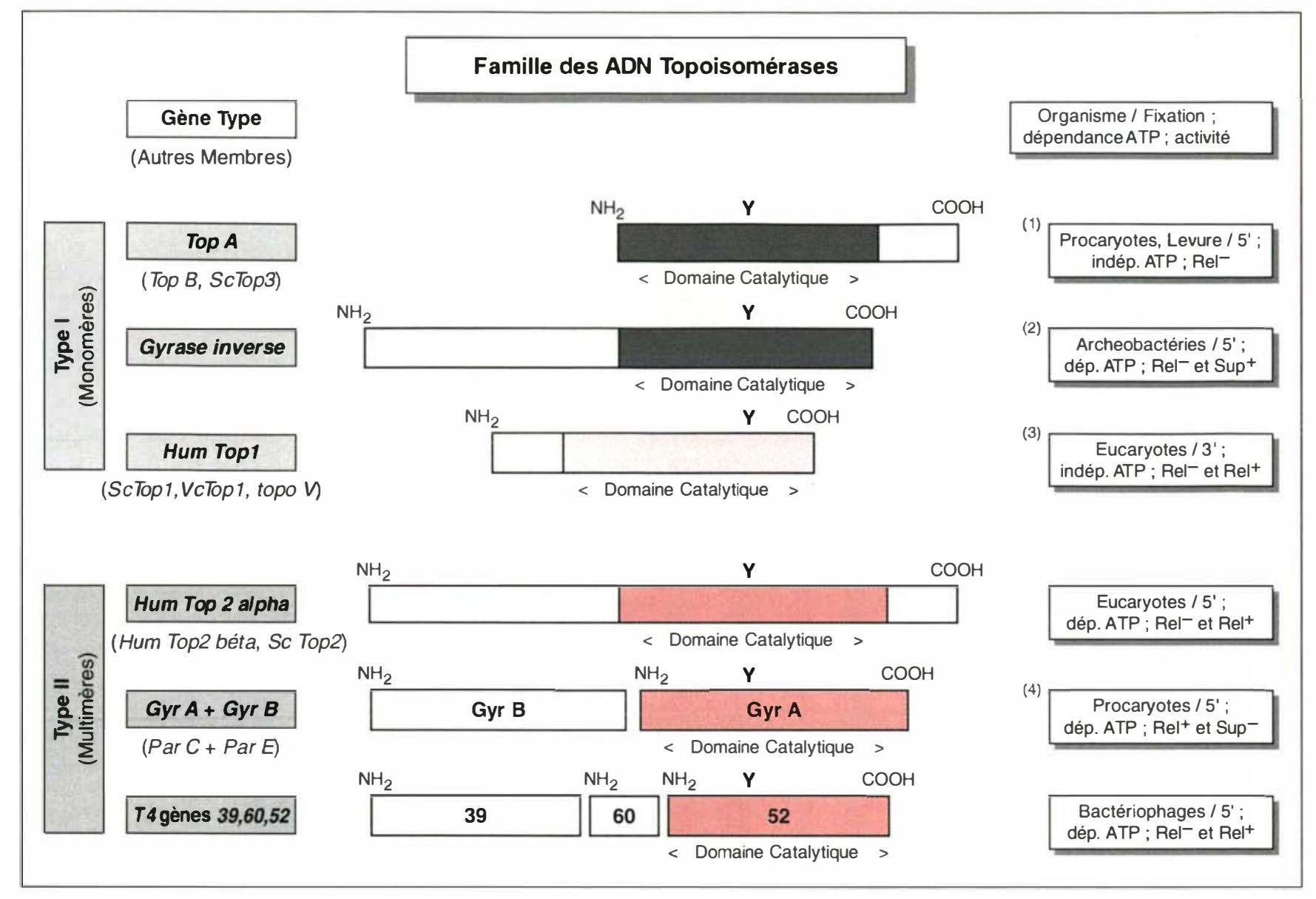

Figure 3. La famille des ADN-topo-isomérases. Les alignements représentés ne sont que qualitatifs : les tailles des polypeptides ne sont pas respectées. Les domaines catalytiques présentant une certaine similarité de séquence sont figurés avec la même convention de teinte (exemple: type II). $\boldsymbol{V}$ indique la tyrosine du site actif. Rel et Rel+ : activité de relaxation des supertours respectivement négatifs et positifs. Sup ${ }^{+}$et sup ${ }^{-}$: activités de surenroulement respectivement négatif et positif. 5' ou 3' indiquent l'extrémité de l'ADN liée de manière covalente à la topo-isomérase. (1) Sc top3 est une topo-isomérase eucaryote (levure S. cerevisiae). (2) La gyrase inverse est également présente chez les eubactéries thermophiles. (3) La topo V est une topo-isomérase archéobactérienne. (4) La topo IV codée par les gènes ParC et ParE n'a pas d'activité de surenroulement.

ce est apparentée [11]. Ce groupe comprend aussi le produit du gène TopB qui code chez E. coli pour la topo-isomérase III, dont la fonction est mal connue [12]. Ces enzymes sont toutes indépendantes de l'ATP, relâchent l'ADN surenroulé négativement, et se fixent à l'extrémité 5' de l'ADN ;

- celui représenté chez les archéobactéries thermophiles par la gyrase inverse (gène Top $R($ ) ), qui existe également chez les eubactéries thermophiles [6, 13]. Elle présente, dans sa partie C-terminale, une similitude de séquence avec le groupe précédent. Les gyrases inverses nécessitent de l'ATP, se fixent en 5', et surenroulent positivement l'ADN ;

- celui représenté par la topo-isomérase I humaine (gène Hum Topl) et dont les membres sont présents chez tous les eucaryotes, chez certains virus, et même chez les archéobactéries [14]. Ce groupe ne présente pratiquement aucune similitude de séquence avec les deux précédents groupes. Il comprend des enzymes indépendantes de l'ATP, à fixation en 3' et qui relâchent les surenroulements positifs et négatifs.

\section{Topo-isomérases de type II}

Contrairement à la précédente, cette famille apparaît assez homogène : toutes les topo-isomérases II présentent des similitudes de séquence, sont multimériques, se fixent en 5', et dépendent généralement de l'ATP. On distingue trois groupes $[8,9]$ :

- celui représenté par la topo-isomé- 


\section{RÉFÉRENCES}

15. Wang JC, Caron PR, Kim RA. The role of DNA topoisomerases in recombination and genome stability: a double-edged sword? Cell 1990 ; $62: 403-6$.

16. Earnshaw WC, Halligan B, Cooke CA Heck MMS, Liu LF. Topoisomerase II is a structural component of mitotic chromosome scaffolds. $\int$ Cell Biol 1985 ; 100 : 1706-15.

17. Cockerill PN, Garrard WT. Chromosomal loop anchorage of the kappa immunoglobulin gene occurs next to the enhancer in a region containing topoisomerase II sites. Cell $1986 ; 44: 273-82$.

18. Adachi $Y$, Luke $M$, Laemmli UK. Chromosome assembly in vitro: topoisome rase $\mathrm{II}$ is required for condensation. Cell $1991 ; 64: 137-48$.

19. Kato JI, Nishimura Y, Imamura R, Niki H, Hiraga S, Suzuki H. New topoisomerase essential for chromosome segregation in $E$ coli. Cell $1990 ; 63$ : 393-404.

20. Drlica K. Bacterial topoisomerases and the control of DNA supercoiling. Trends Genet $1990 ; 6: 433-7$.

21. Saavedra RA. Environmental stimuli and transcriptional activity generate transient changes in DNA torsional tension. Bio Essays 1990; 12 : 125-8.

22. Gilmour DS, Pflufelder G, Wang JC, Lis IT. Topoisomerase I interacts with transcribed regions in drosophila cells. Cell 1986 ; 44 : $401-7$.

23. Merino A, Madden KR, Lane WS, et al. DNA topoisomerase $I$ is involved in both repression and activation of transcription. Nature 1993 ; 365 : 227-32.

24. Kretzschmar M, Meisterernst M, Roeder RG. Identification of human topoisomerase I as a cofactor for activator-dependent transcription by RNA polymerase II Proc Natl A cad Sci USA 1993 ;90: 11508-12.

25. Riou JF, Multon E, Vilarem MJ, Larsen CJ, Riou G. In vivo stimulation by antitumor drugs of the topoisomerase II induced cleavage site in the $c-m y c$ protooncogene. Biochem Biophys Res Commun 1986; 137 : 154-60.

26. Rowe TC, Wang JC, Liu LF. In vivo localization of DNA topoisomerase II cleavage sites on drosophila heat shock chromatin. Mol Cell Biol 1986 ; 6 : 985-92. rase II humaine (gènes TOP2 $\alpha$ et $\beta$ ) qui comprend les enzymes eucaryotes (connues chez les mammifères, les amphibiens, les insectes, les plantes, les champignons...). Les isoformes $\beta$ diffèrent peu de $\alpha$, sauf à l'extrémité C-terminale, plus longue pour $\beta$. Ces enzymes forment et résolvent des caténaires et relâchent l'ADN in vitro;

- celui représenté par la topo-isomérase II d' $E$. coli (gènes GyrA et GyrB), tétramérique $\left(\mathrm{A}_{2} \mathrm{~B}_{2}\right)$ et baptisée gyrase. La gyrase est présente exclusivement chez les bactéries et surenroule négativement l'ADN. Grâce à la similarité des séquences, on peut estimer que les protéines issues des gènes GyrB et GyrA couvrent respectivement les régions $\mathrm{N}$ - et $\mathrm{C}$-terminales des enzymes issues des gènes Top2 eucaryotes (figure 3). Des gènes similaires à GyrA et $B$, dénommés $\operatorname{Par} C$ et $P a r E$, codent, toujours chez les bactéries, pour la topo-isomérase IV ressemblant à la gyrase, mais incapable de surenrouler l'ADN ;

- celui représenté par les topo-isomérases II codées par certains bactériophages: dans le cas du phage T4, l'enzyme est codée par trois gènes dont les séquences couvrent l'ensemble GyrB-GyrA. Elles n'ont pas d'activité de surenroulement, au moins in vitro.

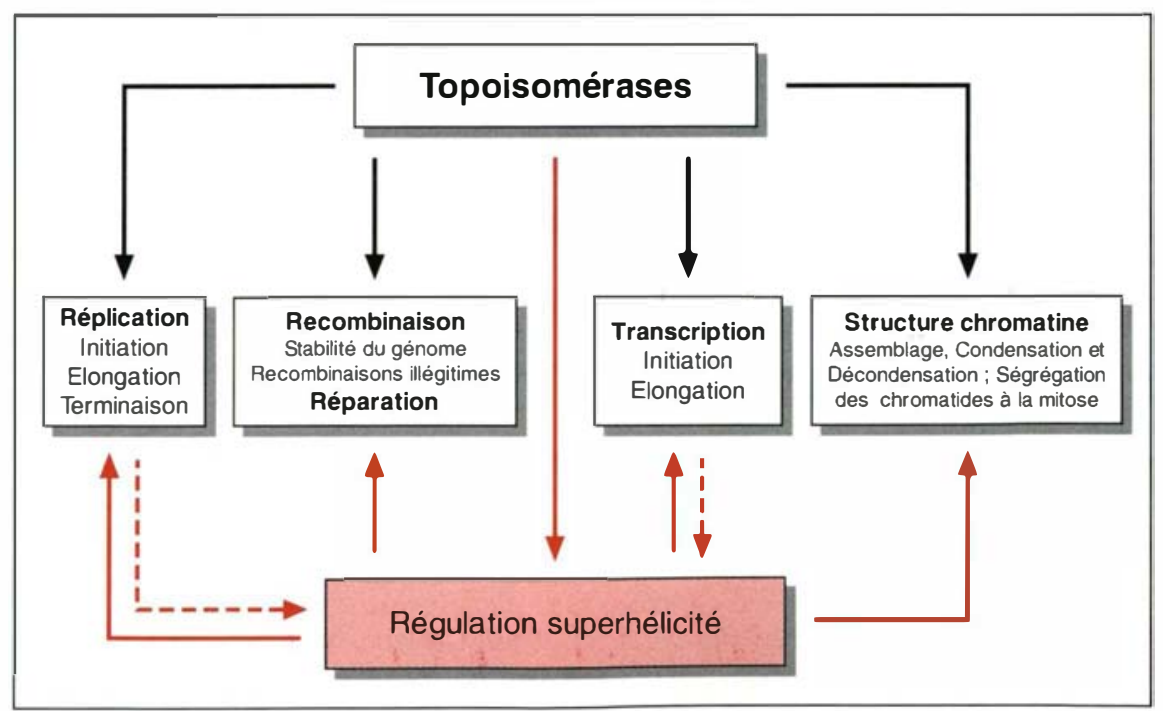

Figure 4. Les topo-isomérases sont nécessaires dans la plupart des processus où I'ADN est impliqué. Elles agissent, soit directement en supprimant les barrières topologiques (flèches noires), soit indirectement en modifiant la superhélicité de l'ADN (flèches rouges). Les flèches discontinues indiquent que la réplication et la transcription sont susceptibles de modifier la superhélicité des génomes.

\section{Les topo-isomérases sont fortement pléiotropes}

Si les topo-isomérases présentent un mécanisme de base commun, elles possèdent, en revanche, des fonctions très variées. On peut distinguer deux rôles majeurs dans la cellule.

(1) Un rôle direct et essentiel qui consiste à éliminer les barrières topologiques au fur et à mesure qu'elles apparaissent, dans tous les processus où l'ADN est impliqué. Ainsi, vont-elles supprimer les contraintes qui sont liées à la progression des fourches de réplication ou des complexes de transcription $[2,4]$. Il est, de même, probable qu'elles sont impliquées dans les appariements des molécules recombinantes et dans le déplacement des jonctions de type Holliday (branch migration) [15]. En outre, chez les eucaryotes, les topo-isomérases, constituants majeurs de la matrice nucléaire, participent activement à l'organisation de la structure chromatinienne [16]. En particulier, la topo-isomérase II possède de nombreux sites de fixation sur l'ADN à la base des boucles de chromatine qui sont ancrées sur la matrice nucléaire [17]. Il semble qu'elle y joue un rôle essentiel dans l'assemblage de la chromatine, dans la condensation et 
Tableau I

LES INHIBITEURS DES ADN TOPO-ISOMÉRASES

\begin{tabular}{|c|c|c|c|c|c|}
\hline & $\begin{array}{l}\text { Agent inhibiteur } \\
\text { (exemple, DCI) }\end{array}$ & $\begin{array}{l}\text { Enzyme } \\
\text { affectée }\end{array}$ & $\begin{array}{l}\text { Étape du } \\
\text { cycle } \\
\text { inhibée }^{\circ}\end{array}$ & $\begin{array}{l}\text { Interaction } \\
\text { avec I'ADN }\end{array}$ & $\begin{array}{l}\text { Activité } \\
\text { biologique }\end{array}$ \\
\hline \multirow[t]{3}{*}{$\begin{array}{l}\text { Procaryotes } \\
\text { Induisant le } \\
\text { complexe de clivage }\end{array}$} & $\begin{array}{c}\text { Quinolones } \\
\text { (Pefloxacine, } \\
\text { Sparfloxacine...) } \\
\end{array}$ & $\begin{array}{l}\text { Gyrase (Gyr A) } \\
\text { ParC, ParE }\end{array}$ & 3 & $\stackrel{+/-}{\left(\mathrm{Mg}^{2+}\right)^{f}}$ & Antibactérien \\
\hline & $\begin{array}{c}\text { Polypeptide } \\
\text { (Microcine B17) }\end{array}$ & Gyrase (Gyr A) & 3 & - & $\begin{array}{l}\text { Activité antibactérienne } \\
\text { in vitro }\end{array}$ \\
\hline & $\begin{array}{l}\text { Terpénoides } \\
\text { (Clerocidine }^{a} \text { ) }\end{array}$ & Gyrase (Gyr A) & 3 & - & $\begin{array}{l}\text { Activité antibactérienne } \\
\text { in vitro }\end{array}$ \\
\hline $\begin{array}{l}\text { N'induisant pas le } \\
\text { complexe de clivage }\end{array}$ & $\begin{array}{l}\text { Coumarines } \\
\text { (Novobiocine) }\end{array}$ & Gyrase (Gyr B) & 4 & - & Antibactérien \\
\hline \multirow[t]{12}{*}{$\begin{array}{l}\text { Eucaryotes } \\
\text { Induisant le } \\
\text { complexe de clivage }\end{array}$} & $\begin{array}{l}\text { Camptothecines } \\
\text { (Irinotecan, } \\
\text { Topotecan) }\end{array}$ & Topo I & 3 & - & $\begin{array}{l}\text { Anticancéreux (études } \\
\text { cliniques de phase II) }\end{array}$ \\
\hline & $\begin{array}{c}\text { Benzo [c] } \\
\text { phénanthridines } \\
\text { (Fagaronine) }\end{array}$ & Topo I & 3 & + & $\begin{array}{l}\text { Activité antileucémique } \\
\text { préclinique }\end{array}$ \\
\hline & $\begin{array}{c}\text { Acridines } \\
\text { (Amsacrine) }\end{array}$ & Topo II & 3 & + & Antileucémique \\
\hline & $\begin{array}{l}\text { Epipodophyl- } \\
\text { lotoxines } \\
\text { (Etoposide, } \\
\text { Teniposide) }\end{array}$ & Topo II & 3 & - & Anticancéreux \\
\hline & $\begin{array}{c}\text { Anthracyclines } \\
\text { (Doxorubicine, } \\
\text { Daunorubicine,...) } \\
\end{array}$ & Topo II & 3 & + & Anticancéreux \\
\hline & $\begin{array}{l}\text { Ellipticines } \\
\text { (Ellipticinium) }\end{array}$ & Topo II & 3 & + & Anticancéreux \\
\hline & $\begin{array}{l}\text { Quinolones } \\
\left(C P-115953^{C}\right)\end{array}$ & Topo II & 3 & $\begin{array}{l}+/- \\
\left(\mathrm{Mg}^{2+}\right)^{4}\end{array}$ & $\begin{array}{c}\text { Activité anticancéreuse } \\
\text { préclinique }\end{array}$ \\
\hline & $\begin{array}{l}\text { Isoflavones } \\
\text { (Génistéine }{ }^{d} \text { ) }\end{array}$ & Topo II & 3 & - & $\begin{array}{l}\text { Activité cytotoxique } \\
\text { in vitro }\end{array}$ \\
\hline & $\begin{array}{l}\text { Benzisoqui- } \\
\text { nolinediones } \\
\text { (Amonafide) }\end{array}$ & Topo ॥ & 3 & + & $\begin{array}{l}\text { Anticancéreux (études } \\
\text { cliniques de phase I) }\end{array}$ \\
\hline & $\begin{array}{l}\text { Actinomycines } \\
\text { (Dactinomycine) }\end{array}$ & Topo I et Topo II & 3 & + & Anticancéreux \\
\hline & $\begin{array}{l}\text { Pyridobenzoindoles } \\
\text { (Intoplicine) }\end{array}$ & Topo I et Topo II & 3 & + & $\begin{array}{l}\text { Anticancéreux (études } \\
\text { cliniques de phase I) }\end{array}$ \\
\hline & $\begin{array}{l}\text { Naphtacene- } \\
\text { 5,8-diones } \\
\text { (Saintopine) }\end{array}$ & Topo I et Topo II & 3 & - & $\begin{array}{l}\text { Activité cytotoxique } \\
\text { in vitro }\end{array}$ \\
\hline \multirow[t]{2}{*}{$\begin{array}{l}\text { N'induisant pas le } \\
\text { complexe de clivage }\end{array}$} & $\begin{array}{l}\text { Barbituriques } \\
\text { (Merbarone) }\end{array}$ & Topo II & 2 & - & $\begin{array}{l}\text { Anticancéreux (études } \\
\text { cliniques de phase I) }\end{array}$ \\
\hline & $\begin{array}{l}\text { Naphtyl-urée } \\
\text { polysulfonate } \\
\text { (Suramined) }\end{array}$ & Topo I et Topo II & 1 & - & $\begin{array}{l}\text { Anticancéreux (études } \\
\text { cliniques de phase I) }\end{array}$ \\
\hline
\end{tabular}

Les différentes classes d'inhibiteurs connues à ce jour sont indiquées par leur nom chimique (quinolones, camptothécines...) et des exemples représentatifs figurent entre parenthèses sous leur dénomination commune intemationale (DCI) (ex. : Pefloxacine, Sparfloxacine). La spérificité du mécanisme d'action est mise en èridence par l'ëtape du cycle inhibee et gräce à une convention de couleurs (blanc, gris clair, gris fonce, rose, rouge) différenciant les stabilisateurs du complexe de clivage des autres classes d'inhibiteurs.

"Induit également le complexe de clivage avec la Topo-isomérase II eucaryote.

${ }^{b}$ Certains dérivés inhibent également la Topo-isomerase II eucaryote.

'Induit aussi le complexe de clivage avec la grase.

"Agit aussi sur d'autres cibles cellulaires.

¿Élape du cycle inhibée: I=inhibileurs de la fixation de la topo-isomerase à l:AI)N; 2=inhibiteurs stabilisant les complexes où l'AIDN n'est pas clivé; $3=s t a b i l i s a t e u r s$ des complexes de clivage; $4=$ inhibiteurs de l'activité ATPase.

Interaction avec liAI) N decrite en l'absence de $M \mathrm{Ig}^{2}$

A noter que les produits ayant une forte interaction avec l'AIN peuvent également inhiber l'étape I lorsqu 'ils sont en concentration éleveee.

$\mathrm{m} / \mathrm{s} n^{\circ} 10$, vol. 10 , octobre 94 


\section{RÉFÉRENCES}

27. Zini N, Martelli AM, Sabatelli P, et al. The $180 \mathrm{kDa}$ isoform of topoisomerase II is localized in the nucleolus and belongs to the structural elements of the nucleolar remnant. Exp Cell Res 1992; 200 : 452-9.

28. Ross WE. DNA topoisomerases as targets for cancer therapy. Biochem Pharmacol i985; $34: 4191-5$

29. Schneider E, Hsiang YH, Liu IF. DNA topoisomerases as anticancer drug targets. Adv Pharmacol 1990 ; 21 : 149-83.

30. Capranico G, Zunino F. DNA topoisomerase-trapping antitumor drugs. Eur J Cancer 1992 ; $28 \AA$ : 2055-60.

31. O'Dwyer PJ, Alonso MT, Letland-Jones $B$, Marsoni $S$. Teniposide : a review of 12 years of experience. Cancer Treat Rep $1984 ; 68: 1455-66$.

32. Weiss RB, Sarosy G, Clagett-Carr K. Anthracycline analogs : the past, present and future. Cancer Chemother Pharmacol $1986 ; 18: 185-97$

33. Slichenmyer WJ, Rowinski EK, Donehower RC, Kaufmann SH. The current status of camptothecin analogues as antitumor agents. Natl Cancer Inst 1993 ; $85: 271-91$.

34. Fossé P, Charra M, Paoletti C, Saucier JM, René B. Stimulation in vitro par des dérivés de l'ellipticine des coupures de l'ADN induites par l'ADN topoisomerase II : relation structure-activité. Bull Cancer $1994 ; 81$ : 194-202.

35. Pommier Y, Kohn KW, Capranico G, Jaxel C. Base sequence selectivity of topoisomerase inhibitors suggests a common model for drug action. In : Andoh T, Ikeda M, Oguro M, eds. Molecular biology of DNA topoisomerases and its application to chemotherapy. Boca Raton : CRC Press, 1993 : 215-27.

36. Gewirtz DA. Does bulk damage to DNA explain the cytostatic and cytotoxic effects of topoisomerase II inhibitors? Biochem Pharmacol 1991 ; 42 : 2253-8.

37. Morrow CS, Cowan KH. Multidrug resistance associated with altered topoisomerase II activity-topoisomerase II as targets for rational drug design. I Natl Cancer Inst $1990 ; 82$ : 638-9.

38. Smith PJ. DNA topoisomerase dysfunction: a new goal for antitumor chemotherapy. BioEssays $1990 ; 12$ : 167-72.

39. Giovanella BC, Stehlin JS, Wall ME, et al. DNA topoisomerase I-targetted chemotherapy of human colon cancer in xenografts. Science 1990 ; 246 : 1046-51

40. Holden JA, Rolfson DH, Wittwer CT Human DNA topoisomerase II : evaluation of enzyme activity in normal and neoplastic tissues. Biochemistry $1990 ; 29: 2127-34$. la décondensation des chromosomes au cours du cycle cellulaire et la ségrégation des molécules d'ADN filles lors de la mitose* [18].

De même, chez les bactéries, la topoisomérase IV (codée par les gènes ParC, Park) serait impliquée, grâce à sa localisation membranaire, dans l'ancrage du chromosome bactérien, dans son organisation en domaines (boucles) distincts et dans la ségrégation des molécules en fin de réplication [19].

(2) Les topo-isomérases jouent un rôle indirect et plus subtil dans le contrôle de l'état énergétique de la molécule d'ADN grâce à la régulation homéostatique de son niveau de surenroulement in vivo (figure 4). Celui-ci résulte, soit de l'activité directe de certaines topo-isomérases (gyrase, gyrase inverse chez les procaryotes) [20], soit des divers stress engendrés dans l'ADN par la progression des complexes de réplication ou de transcription et qui sont modulés par l'activité des topo-isomérases [21]. A son tour, le niveau de surenroulement de l'ADN est capable d'exercer un effet régulateur des processus de réplication, de transcription et de recombinaison, notamment au niveau de leur initiation [8]. Ainsi, l'implication de la topo-isomérase I dans la progression des complexes de transcription se traduit-elle par une localisation préférentielle de cette enzyme au niveau des gènes activement transcrits [22]. Deux travaux démontrent l'association directe de la topo-isomérase I au complexe de transcription en tant quactivateur ou même répresseur [23, 24]. La fonction précise de la topo-isomérase I dans ce complexe demeure cependant inconnue, puisque la répression de la transcription ne nécessite même pas une topo-isomérase active [23]. Quant à la topo-isomérase II, son rôle dans l'initiation de la réplication est illustré par la localisation de nombreux sites de fixation de cette enzyme au niveau des régions régulatrices et promotrices de divers gènes $[25,26]$. De plus, la présence

\footnotetext{
* Serules les topo-isomérases de type II sont capables de ségréger les AIDN bicaténaires fennés, erâce ì leur activité de destruction des caténaires.
}

de l'isoenzyme $\beta$ de la topo-isomérase II dans la matrice nucléolaire suggère que l'enzyme est impliquée dans la transcription des ADN ribosomiques [27]. Enfin, l'influence de la superhélicité (état topologique) de l'ADN sur le taux de recombinaison est démontrée dans divers systèmes, sans que les mécanismes en soient bien compris [15].

\section{Les inhibiteurs des topo- isomérases : antibiotiques et antitumoraux}

Dans les années 1980, bon nombre d'anticancéreux (acridines, actinomycines, anthracyclines, ellipticines, épipodophyllotoxines, camptothécines...) et deux classes d'antibactériens (quinolones, coumarines) ont vu leur mécanisme d'action expliqué par leur capacité d'inhiber les topoisomérases (Tableau I) [28-30]. On distingue classiquement deux types d'inhibiteurs, ceux qui inhibent les enzymes procaryotes (gyrases) et ceux qui inhibent les enzymes eucaryotes (topo-isomérases I et II). Cette distinction souffre plusieurs exceptions, notamment dans le cas de la clérocidine et de certaines quinolones (CP-115953) qui sont actives à la fois sur la gyrase et sur la topoisomérase II eucaryote. Les inhibiteurs de la topo-isomérase II, tels que le téniposide ou la doxorubicine, sont utilisés en clinique contre plusieurs types de cancer comme ceux du sein, du poumon, du testicule, certaines tumeurs pédiatriques (lymphomes, leucémies...) [31, 32].

Récemment, les camptothécines ont été caractérisées comme inhibiteurs de la topo-isomérase I eucaryote [ 29 , 30]. Plusieurs dérivés hydrosolubles de ces agents font l'objet d'études cliniques de phase II et montrent des activités prometteuses dans le traitement du cancer du côlon [33]. En revanche, aucun inhibiteur de la topo-isomérase I procaryote n'est connu actuellement.

Enfin, certains agents anticancéreux inhibent à la fois les topo-isomérases I et II. C'est le cas de l'actinomycine $\mathrm{D}$, de l'intoplicine, de la saintopine, et de certains dérivés de la fagaronine (Tableau I). En dehors de la classification chimique et de la spécificité de ces inhibiteurs, on peut, d'un point de vue fonctionnel, 
caractériser plusieurs types d'inhibiteurs par l'étape du cycle de topoisomérisation (figure 2) qui est impliquée. On peut, en effet, considérer a priori que le bloquage de n'importe quelle étape intermédiaire de la réaction provoque l'inhibition de l'activité catalytique. On distingue (1) les inhibiteurs de la fixation de la topo-isomérase à l'ADN. Ces produits peuvent agir, soit sur la topoisomérase (suramine), soit sur l'ADN (cas des intercalants); (2) les inhibiteurs de la dissociation de la topoisomérase de l'ADN qui agissent en stabilisant les complexes où l'ADN n'est pas clivé (merbarone) ; (3) les inhibiteurs de l'activité ATPase (cas de la topo-isomérase II) qui inhibent la régénération de l'enzyme nécessaire au cycle catalytique suivant (coumarines, orthovanadate); (4) les stabilisateurs des complexes de clivage. Ces agents stabilisent les intermédiaires réactionnels fugaces dans lesquels l'ADN est clivé et fixé de façon covalente à la topo-isomérase (complexes de clivage). L'accumulation de tels complexes résulte, soit d'une stimulation de l'étape de clivage, soit le plus souvent d'une inhibition de l'étape de religation (figure 2). ('ette classe d'inhibiteurs, les plus nombreux (Tableau I), présente un intérêt majeur dans la mesure où la cytotoxicité semble liée à l'accumulation des complexes de clivage (voir ci-après). Ces agents présentent une grande hétérogénéité de structure chimique qui se traduit par de grandes différences dans leurs propriétés de fixation à l'ADN. L'étude des relations structure-activité de ces inhibiteurs permet de définir certains groupements chimiques importants pour l'inhibition de la topo-isomérase, comme par exemple l'hydroxyle en position 9 pour les dérivés de l'ellipticine [34], mais ne permet pas de définir, au sens strict, un pharmacophore commun [28-31, 33]. Certains de ces agents ont une interaction forte avec l'ADN et peuvent détordre une molécule d'ADN in vitro. C'est le cas des intercalants qui produisent un effet biphasique : à faible concentration, ils induisent la formation des complexes de clivage, cependant qu'ils l'inhibent à plus forte concentration, en empêchant la fixation de la topo-isomérase à l'ADN. La stabilisation du com$\mathrm{m} / \mathrm{s} n^{\circ} 10$, vol. 10, octobre 94 plexe de clivage présente une certaine spécificité vis-à-vis de la séquence, certaines bases étant préférées au niveau du site de clivage. Cette préférence varie d'un agent à un autre [35]. On peut imaginer un modèle d'interaction dans lequel la réaction de religation est inhibée par le positionnement de l'agent au centre de la région où la topo-isomérase interagit avec l'ADN et le clive. Le groupe $\mathrm{OH}$ de l'extrémité du brin clivé serait placé dans une position trop éloignée du site actif pour que la réaction de religation (trans-estérification) puisse se faire [29].

\section{Comment l'action des inhibiteurs des topo- isomérases conduit-elle à la cytotoxicité ?}

Le concept de complexe de clivage comme lésion potentiellement létale

Dans la mesure où les topo-isomérases sont essentielles à la plupart des mécanismes génétiques, il était logique de penser que l'inhibition pure et simple de ces enzymes pouvait être létale pour la cellule. Il est clair maintenant que la cytotoxicité n'est pas directement corrélée au pouvoir inhibiteur de tel ou tel agent [36]. Cela est bien illustré par les résultats concernant les cellules résistantes aux inhibiteurs des topoisomérases: on s'attend, comme dans tous les cas classiques de résistance à un inhibiteur, à ce qu'une augmentation de la quantité de topo-isomérase cible se traduise par une résistance accrue à l'inhibiteur. C'est l'inverse qui se produit: les cellules résistantes ont un contenu plus faible en topo-isomérases, cependant que les cellules ayant un contenu élevé en topo-isomérases sont hypersensibles [37, 38] ! La clé de ce paradoxe réside dans l'idée que la cytotoxicité est liée à l'accumulation de complexes stables "inhibiteur - topo-isomérase ADN » dans lesquels l'ADN est clivé (complexes de clivage): plus il y a de topo-isomérase au voisinage de l'ADN, plus ces complexes se forment en grand nombre sur l'ADN, et plus grande est la cytotoxicité. C'est également ce qui explique la relative sélectivité des agents vis-à-vis des cellules tumorales, qui ont un contenu élevé en topo-isomérases, par rapport aux cellules normales $[39,40]$.

\section{Du complexe de clivage à la mort cellulaire}

Le traitement de la cellule par divers inhibiteurs des topo-isomérases se traduit par la formation de complexes de clivage dans l'ADN génomique : on peut obtenir un "instantané " de la répartition de ces complexes par différentes techniques (élution alcaline, Southern blot...). Cependant, les complexes de clivage disparaissent dès que l'on retire l'agent et les lésions produites sont donc réversibles [29]. Ces complexes sont-ils létaux? Un grand nombre de travaux indiquent que la corrélation entre complexe de clivage et cytotoxicité est très variable et dépend de l'inhibiteur utilisé. Par exemple, la doxorubicine et l'intoplicine induisent peu de complexes de clivage dans la cellule et sont aussi cytotoxiques que l'étoposide ou l'amsacrine qui en induisent dix à vingt fois plus. De nombreux paramètres semblent entrer en ligne de compte pour moduler l'activité cytotoxique de ces inhibiteurs [41].

- L'efficacité relative de pénétration et d'efflux de l'agent et sa possible métabolisation selon le type cellulaire ; cela va déterminer la concentration effective de l'agent au niveau de la cible: par exemple, certains inhibiteurs de la topo-isomérase, tels les camptothécines, ne sont pas reconnus par le système d'excrétion de la P-glycoprotéine (gène $M D R$ ), ce qui peut expliquer l'activité clinique de ce dérivé dans les tumeurs coliques exprimant naturellement le gène $M D R[42]$.

- La stabilité de l'interaction avec la cible (topo-isomérase - ADN) qui détermine le temps de résidence des lésions dans la cellule : par exemple, ce temps est long dans le cas de la doxorubicine et de l'intoplicine (peut-être parce qu'elles ont une forte interaction avec l'ADN) et court dans le cas de l'étoposide et de l'amsacrine.

- La quantité de topo-isomérase, très variable d'un type cellulaire à l'autre, qui conditionne directement le nombre de lésions possibles 


\section{RÉFÉRENCES}

41. Pommier Y, Leteurtre F, Fesen MR, et al. Cellular determinants of sensibility and resistance to topoisomerase inhibitors. Cancer Commun 1994 (sous presse).

42. Tsurno T, Matsuzaki T, Matsushita M, Saito H, YokokuraT. Antitumor effect of CPT 11, a new derivative of camptothecin, against pleiotropic drug-resistant tumors in vitro and in vivo. Cancer Chemother Pharmacol $1988 ; 21: 71-4$.

43. Zhang H, D'Arpa P, Liu IF. A model of tumor cell killing by topoisomerase poisons. Cancer Cells $1990 ; 2$ : 23-7.

44. Charron M, Hancock R. Chromosome recombination and defective genome segregation induced in Chinese hamster cells by the topoisomerase II inhibitor VM26. Chromosoma 1991 ; 100 : 97-102.

45. Golstein P. Mort programmée et terrain cellulaire. médecine/sciences 1991 ; 7 : 681-8.

46. Solary E, Bertrand R, Pommier Y. Le rôle de l'apoptose dans la genèse et le traitement du cancer. médecine/sciences 1993 ; $9: 667-75$.

47. Downes CS, Johnson RT. DNA topoisomerases and DNA repair. Bio Essays 1988 ; $8: 179-84$.

48. Nitiss JL, Liu YX, Hsiung Y. A temperature sensitive topoisomerase II allele confers temperature dependent drug resistance on amsacrine and etoposide : a genetic system for determining the targets of topoisomerase II inhibitors. Cancer Res $1993 ; 53: 89-93$

49. Squires S, Ryan AJ, Strutt HL, Johnson RT. Hypersensitivity of Cockayne's syndrome cells to camptothecin is associated with the generation of abnormally high levels of double strand breaks in nascent DNA. Cancer Res 1993 ; 53 : 2012-9.

50. Sarasin A. Les gènes humains de la réparation de l'ADN. médecine/sciences $1994 ; 10: 43-54$

51. Wigley DB, Davies GJ, Dodson EJ, Maxwell A, Dodson G. Crystal structure of an N-terminal fragment of the DNA gyrase B protein. Nature 1991 ; 351 : 624-9.

52. Lima C, Wang JC, Mondragon A. Three dimensional structure of the $67 \mathrm{~K}$ N-terminal fragment of $E$. coli DNA topoisomerase I. Nature $1994 ; 367$ : 138-46.

53. Pommier Y, Orr A, Kohn K, Riou JF. Differential effects of amsacrine and epipodophyllotoxins on topoisomerase II cleavage in the human $c-m y c$ protooncogene. Cancer Res $1992 ; 52: 312530$.

54. Woessner RD, Mattern MR, Mirabelli CK, et al. Proliferation- and cell cycledependent differences in expression of the 170 kilodalton and 180 kilodalton forms of topoisomerase II in NIH 3T3 cells. Cell comme indiqué précédemment, l'un des mécanismes de résistance aux inhibiteurs de topo-isomérases est la diminution de la quantité de ces enzymes dans la cellule.

- Le moment du cycle cellulaire où l'agent est appliqué : ce paramètre semble jouer un rôle primordial ; bon nombre de médicaments, notamment la camptothécine, sont particulièrement cytotoxiques lorsqu'ils sont appliqués en phase S [43]. - Enfin, comme indiqué dans le paragraphe précédent, la formation de complexes de clivage est partiellement dépendante de la séquence d'ADN, au moins in vitro, et différents inhibiteurs sélectionnent des régions de clivage (toposites) différentes [35]. Cela pourrait exister également in vivo, malgré la présence de la structure chromatinienne. Ainsi, au niveau de gènes particuliers (c'est le cas de C-MYC), la localisation des toposites dépend de l'agent [25]. Le type de région touchée (parties codantes de gènes/espaces intergéniques) pourrait, lui aussi, jouer un rôle dans l'importance létale de la lésion [36]. Les considérations précédentes traduisent le fait qu'en réalité la formation des complexes de clivage est le premier d'une longue série d'événements encore mal connus et qui conduisent à la mort cellulaire (figure 5). Cette idée est illustrée par le fait que la cytotoxicité des inhibiteurs de topo-isomérases est considérablement réduite en présence de divers inhibiteurs de la synthèse des protéines ou des ARN [43]

Comme schématisé sur la figure 5, la réplication, la transcription, et peutêtre d'autres processus comme la recombinaison pourraient transformer les complexes initiaux en lésions secondaires dans l'ADN. Un exemple frappant est celui de la camptothécine qui induit un grand nombre de complexes de clivage avec la topo-isomérase I. Les complexes, dans lesquels un seul brin est clivé, ne sont cytotoxiques que si la cellule est en phase $S$ : il a été proposé que la collision des fourches de réplication avec les complexes produits des cassures double-brin irréversibles qui sont, finalement, cytotoxiques [43]. Par ailleurs, des aberrations chromosomiques nombreuses sont observées dans les cel- lules traitées par les inhibiteurs de topo-isomérases, tels des échanges de chromatides sœurs et des recombinaisons illégitimes [44]. Ces anomalies peuvent être la conséquence directe (recombinaison par échange de brins) ou indirecte (réparation anarchique) de la présence de complexes de clivage.

Au stade des lésions secondaires (figure 5), deux possibilités s'offrent : ou bien l'ADN est réparé, et la cellule est sauvée, ou bien la lésion persiste et la cellule meurt par apoptose ou autres processus. L'apoptose, ou mort cellulaire programmée [45], est un phénomène naturel qui va de pair avec la prolifération pour maintenir l'homéostasie tissulaire: il agit dès les premiers stades du développement embryonnaire et est soigneusement réglé. Sur un plan morphologique, l'apoptose se traduit, entre autres, par une condensation de la chromatine suivie d'une fragmentation du noyau, et, sur le plan biochimique, par une fragmentation de l'ADN à la taille nucléosomiale. Récemment, l'hypothèse a été avancée que l'apparition des cancers pouvait être liée à une dérégulation de l'apoptose [46]

Quoi qu'il en soit, plusieurs travaux indiquent que les inhibiteurs des topo-isomérases induisent la mort par apoptose dans diverses cellules tumorales ou normales en prolifération. Les mécanismes de réparation semblent jouer un rôle important dans l'aiguillage de la cellule vers la mort cellulaire ou vers la non-létalité des lésions. Ainsi, chez les bactéries, les quinolones induisent très efficacement le système SOS [47]. Chez la levure, l'inactivation du gène $R A D 52$, nécessaire à la réparation des cassures double-brin, provoque une hypersensibilité aux inhibiteurs des topo-isomérases [48]. La même hypersensibilité est observée dans des lignées cellulaires humaines déficientes en réparation (ataxie-télangiectasie, SCID (severe combined immunodeficiency), syndrome de Cockayne...) [47-50].

\section{Perspectives thérapeutiques}

La chimiothérapie antitumorale, antibactérienne et antivirale est, à l'heure actuelle, encore très empi- 


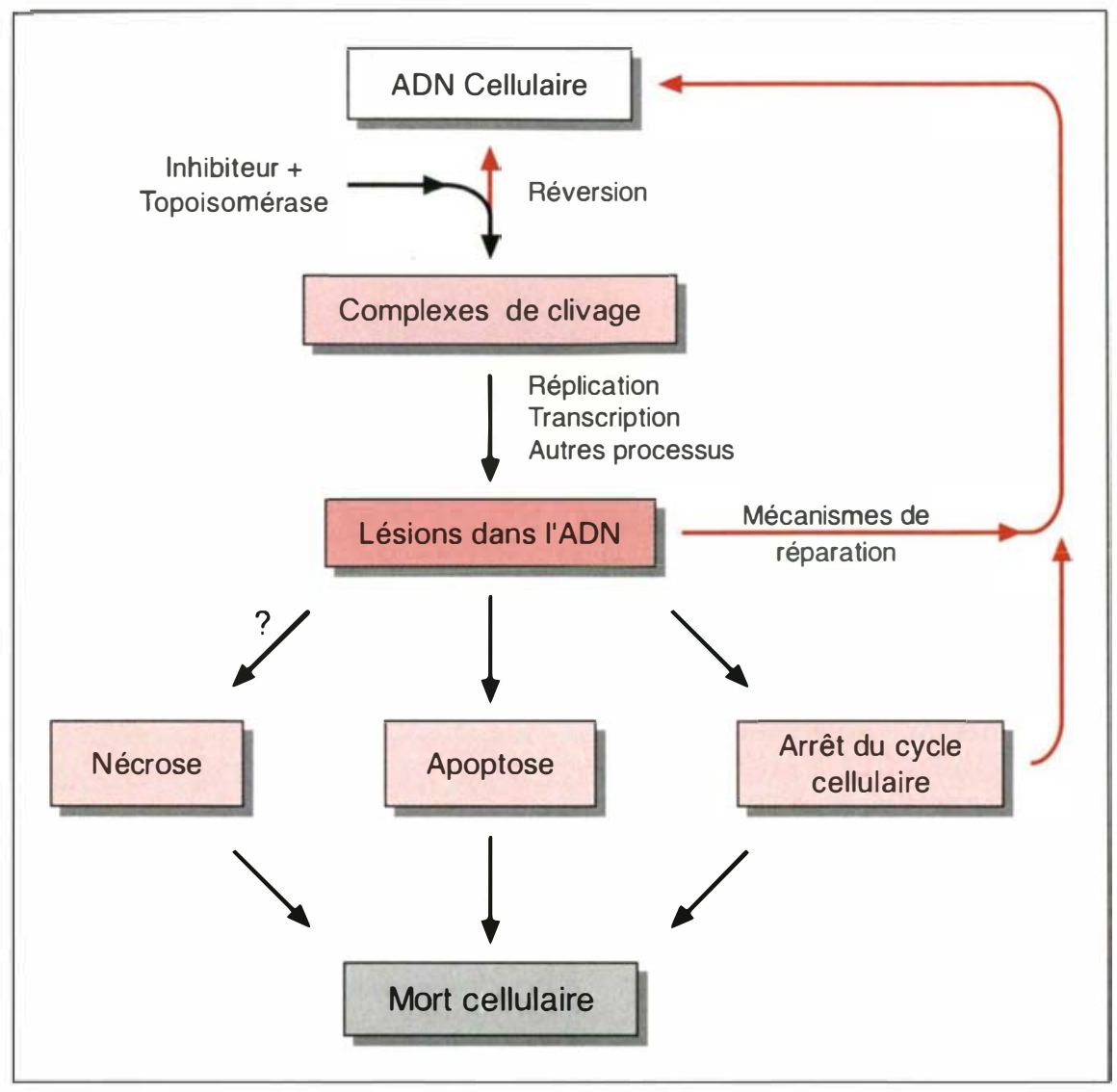

Figure 5. Des complexes de clivage à la mort cellulaire. Le point d'interrogation indique que la mort cellulaire par nécrose après un traitement par les inhibiteurs de topo-isomérases n'est pas démontrée.

rique et tout, ou presque, reste à faire. On peut ainsi améliorer la pénétration du médicament jusqu'à sa cible, son efficacité à tuer les cellules tumorales ou bactériennes, sa sélectivité vis-à-vis de ces cellules, et contrecarrer l'apparition de cellules résistantes. L'étude des nouvelles topo-isomérases permettra aussi d'élargir le domaine d'application thérapeutique de leurs inhibiteurs.

\section{Synthèse rationnelle de nouveaux composés}

Jusqu'à présent, l'identification des topo-isomérases comme une cible majeure de nombreux antitumoraux et antibiotiques a permis, par l'étude des relations structure-activité, de définir les groupes chimiques importants pour l'activité des inhibiteurs $\mathrm{m} / \mathrm{s} n^{\circ} 10$, vol. 10, octobre 94 d'un fragment de la topo-isomérase I (Top A) bactérienne [52].

Cibler des gènes spécifiques : vers des traitements personnalisés

On sait que les cellules tumorales présentent un programme génétique profondément remanié. Elles expriment des gènes qui ne sont pas exprimés dans les cellules normales correspondantes (marqueurs embryonnaires, gènes impliqués dans la croissance autocrine, y compris certains oncogènes...). L'une des perspectives thérapeutiques serait de toucher spécifiquement des gènes essentiels au fonctionnement et à la croissance des cellules tumorales. Cette perspective n'est plus hors de portée, puisque l'on sait que différents antitumoraux touchent plus ou moins spécifiquement différents gènes, particulièrement ceux qui sont activement exprimés, tel le gène C-MYC [53]. L'enjeu consiste précisément à accroître cette spécificité, en jouant par exemple sur l'interaction de l'inhibiteur avec la base de l'ADN proche du point de clivage de l'enzyme et sur le choix de la séquence d'ADN à cibler (oncogène). Les avantages sont ceux déjà évoqués : efficacité plus grande, sélectivité réelle pour les cellules tumorales, réduction des effets secondaires. De plus, on peut imaginer un traitement adapté à chaque patient après une analyse du programme d'expression des cellules de la tumeur dont il souffre, ou, plus généralement, en fonction des gènes exprimés dans une maladie tumorale déterminée.

Agir sur les circuits naturels conduisant à la cytotoxicité

Il s'agit d'accroître l'efficacité avec laquelle les complexes de clivage induisent la mort cellulaire. Cette perspective attrayante est plus lointaine, dans la mesure où les mécanismes de régulation sont mal connus. Dans le cas de l'apoptose, on commence à entrevoir ces mécanismes, avec la mise en évidence du rôle des produits des gènes $C-M Y C$ et $B C L-2$, et de la protéine p53. On ne connaît cependant pas la nature du ou des signaux par lesquels les complexes de clivage induisent l'apoptose. 


\section{Le problème des cellules résistantes}

En dehors des circuits permettant de bloquer l'activité du gène $M D R$ (protéine gp 170 impliquée dans la résistance à de multiples médicaments par augmentation de l'efflux), il existe de nombreux cas de résistance croisée aux inhibiteurs des topo-isomérases. Il s'agit, soit d'une modification quantitative de la cible (diminution de l'expression de la topo-isomérase), soit d'une altération de l'activité de l'enzyme par des modifications post-traductionnelles (phosphorylation) ou par des mutations ponctuelles [41]. Dans ce dernier cas, on peut imaginer l'élimination des cellules résistantes en utilisant un nouvel inhibiteur non " reconnu " par la mutation.

\section{Nouvelles topo-isomérases : élargis- sement du potentiel thérapeutique}

Les topo-isomérases de parasites (trypanosome, Plasmodium), de levures pathogènes (Candida), de virus (Herpes, VIH), ou même de plantes, sont sensibles in vitro à certains des agents antitumoraux décrits précédemment (acridines, ellipticines, épipodophyllotoxines, et camptothécines). L'obtention de dérivés plus spécifiquement actifs sur ces topoisomérases peut conduire dans un avenir proche à un élargissement du "potentiel thérapeutique " des topoisomérases.

De plus, aucune topo-isomérase I du groupe de Top A (Top A, Top B, Sc Top 3, voir figure 3) n'a d'inhibiteurs connus à ce jour. Pourtant, de tels inhibiteurs devraient posséder une activité bactéricide intéressante. La détermination de la structure cristalline de TopA pourrait permettre la mise au point de tels inhibiteurs.

Enfin, la redondance des topo-isomérases de type II aussi bien chez les bactéries (gyrase et topo IV) que chez les mammifères (variants $\alpha$ et $\beta)$ offre également quelques perspectives de sélectivité, s'il se confirme que chaque variant présente une sensibilité différente aux agents et n'est pas spécifique de la même phase du cycle cellulaire [54].

\section{Conclusion}

Parmi les nombreuses approches thérapeutiques envisagées pour le traitement des cancers et des maladies infectieuses, la voie des topo-isomérases, remarquablement peu exploitée jusqu'à présent*, apparaît comme une possibilité prometteuse. Le dysfonctionnement de ces enzymes, qui sont au "cœur" du métabolisme de l'ADN, a en effet des conséquences dramatiques sur la vie de la cellule. On peut imaginer, en particulier à l'aide de l'outil de la modélisation moléculaire, la mise au point de molécules infiniment plus performantes, non seulement par leur simple efficacité à tuer les cellules indésirables, mais plutôt par leur extrême spécificité vis-à-vis de ces cellules. Ces perspectives devraient constituer, avec l'approche de la thérapie génique et les nouvelles cibles que constituent les oncogènes et les gènes suppresseurs, une avancée décisive en santé publique, dans les traitements des cancers, et des agents pathogènes comme les bactéries, les parasites, et les virus

\section{Remerciements}

Nous adressons nos sincères remerciements aux Dr C. Combeau et F. Lavelle (Rhône-Poulenc Rorer), V. Borde, C. Bouthier de la Tour, F. Confalonieri, A-C Declais, C. Esnault, C. Jaxel, M. Nadal (laboratoire d'enzymologie des AN Orsay) pour leur lecture critique du manuscrit, et le $\mathrm{Pr}$ Neil Osheroff (Vanderbilt University, Nashville, Tennessee) pour l'autorisation de reproduire son schéma du mécanisme de la topo-isomérase II. Le laboratoire d'enzymologie des acides nucléiques est soutenu par des fonds de l'ARC.

\section{Summary}

From DNA topology to clinical active antibiotics and antitumor agents

The control of DNA topology and the multiple processes linked to DNA metabolism, i.e. gene expression, replication or recombination, are achieved by specialized enzymes named DNA topoisomerases. These ubiquitous enzymes form a fast growing family of proteins with a common basic mechanism : they are all able to produce transient breaks in the DNA backbone and to allow the passage of DNA segments through these breaks before resealing. They are usually divided into two classes : type I topoisomerases produce transient single-strand breaks, while type II topoisomerases can simultaneously cleave both strands. These latter enzymes form a rather homogeneous group of proteins by their biochemical properties as well as their sequence similarities. On the contrary, type I topoisomerases are heterogeneous, with important differences in their structural and biochemical properties, depending on their archaebacterial, eubacterial or eukaryotic origin. DNA topoisomerases (I or II) are the intracellular targets of several classes of antitumor or antibacterial agents. Knowing the precise modes of interaction of these compounds with topoisomerases and DNA would permit to better understand the basis of their activity. In addition, the long pathway from production of lesions in the DNA molecule to cell death begins to be elucidated. These studies open promising developments for the rational design of new antitumor, antibacterial and antiviral agents with considerably more selective and more powerful activities.

TIRÉS A PART

J.-F. Riou.
* Même si divers inhibiteurs de topo-isomérases sont utilisés depuis longtemps de manière empirique. 